UAB-FT-366

May 1995

\title{
THE ORTHOPOSITRONIUM DECAY PUZZLE AND PRIMORDIAL NUCLEOSYNTHESIS
}

\author{
R. Escribano, E. Massó AND R. TOLDRÀ \\ Grup de Física Teòrica and Institut de Física d'Altes Energies \\ Universitat Autònoma de Barcelona \\ 08193 Bellaterra, Barcelona, Spain
}

\begin{abstract}
The discrepancy between the experimental decay rate of orthopositronium (o-Ps) and the QED theoretical prediction can be solved by invoking decays of o-Ps into exotic particles with branching ratios $\sim 10^{-3}$. We show that considerations based on primordial nucleosynthesis and effective Lagrangians place a very stringent upper bound: $B \equiv \Gamma(\mathrm{o}-\mathrm{Ps} \rightarrow$ "exotic" $+\ldots) / \Gamma(\mathrm{o}-\mathrm{Ps}) \leq 2 \times 10^{-15}$, ruling out the exotic decay solution to the puzzle.
\end{abstract}




\section{Introduction}

In the last few years, experiments [1, [] performed on the orthopositronium (o-Ps) system $-\mathrm{e}^{+} \mathrm{e}^{-}$bound in the ${ }^{3} \mathrm{~S}_{1}$ state- have shown that its decay rate in vacuum is significantly higher than the QED prediction. The data obtained by Westbrook et al. [1], give $\lambda_{\exp }=$ $7.0516 \pm 0.0013 \mu \mathrm{s}^{-1}$, while Nico et al. [2] obtain $\lambda_{\text {exp }}=7.0482 \pm 0.0016 \mu \mathrm{s}^{-1}$. However, the QED expectation [3] is $\lambda_{t h}=7.03830 \pm 0.00007 \mu \mathrm{s}^{-1}$, so that there is a significant discrepancy between theory and experiment.

One of the proposed solutions to this problem is that, apart from the standard $3 \gamma$ decay, there are new disintegration channels of o-Ps. Indeed, if o-Ps has exotic decays, with a branching ratio on the order of $10^{-3}$, the theoretical prediction would increase in the right amount to be consistent with experiment.

Much attention has been devoted to decays with final states that involve exotic particles. The decay mode o-Ps $\rightarrow \gamma X$, where $X$ is a weakly interacting particle [4], or the decay into "invisible" final states, could solve the orthopositronium decay rate puzzle. Several groups have been searching for such new decays of o-Ps [5]- [9]. The most restrictive experimental results on these exotic decays that have been published are:

- o-Ps $\rightarrow \gamma X$ has, if $X$ is short-lived, the following upper limits on the branching ratios: $2.0 \times 10^{-4}$ for $847 \mathrm{keV} \leq m_{X} \leq 1013 \mathrm{keV}$ [5], $3.0 \times 10^{-4}$ for $m_{X} \leq 500 \mathrm{keV}$ [6]

- o-Ps $\rightarrow \gamma X$ has, if $X$ is long-lived, a branching ratio that is less than $1.1 \times 10^{-6}$, provided $m_{X} \leq 800 \mathrm{keV}$ [目]

- o-Ps $\rightarrow$ "nothing", has also been searched for. The absence of any "invisible" event gives a branching ratio less than $2.8 \times 10^{-6}$ [ 8 .

We see that the decay o-Ps $\rightarrow$ "nothing" is ruled out as an explanation of the o-Ps anomaly, and that the decay o-Ps $\rightarrow \gamma X$ is excluded in most of the possible $X$ mass range, $0 \leq m_{X} \leq 1022 \mathrm{keV}$, but not in all the range.

The experimental limit on o-Ps $\rightarrow \gamma X$ is based on the search for a monochromatic photon in the final state. We note that a decay such as o-Ps $\rightarrow \gamma X_{1} X_{2}$, where there are now two exotics in the final state could provide a solution for the o-Ps puzzle, but there are no experimental limits on such decays, the reason being that now the photon is not monochromatic.

In this letter we place bounds on the o-Ps exotic decays based on primordial nucleosynthesis arguments. We will obtain much more restrictive bounds than the experimental results, for any decay of o-Ps into final states containing at least one exotic particle. 
Simply stated, our idea is the following. A exotic particle that is produced in o-Ps decay is coupled to electrons, positrons and/or to photons with a strength that will determine the branching ratio for this mode. In the early Universe, that particle will be maintained in thermal equilibrium due to its exotic coupling to $\mathrm{e}^{+}, \mathrm{e}^{-}$and/or $\gamma$. However, the exotic particle must decouple by the nucleosynthesis era $(T \sim 1 \mathrm{MeV})$ since otherwise the successful predictions of the standard primordial nucleosynthesis would be spoiled. This requirement places strong limits on the exotic coupling, and consequently on the exotic decay of o-Ps.

We are interested in the contribution to the effective degrees of freedom of new particles with masses up to the o-Ps mass, i.e., $0 \leq m \leq 1022 \mathrm{keV}$. Therefore, in general, we cannot neglect the mass $m$ compared to the temperatures $T$ that we will have to consider, since the latter go down to $T \sim 1 \mathrm{MeV}$. We will discuss how to treat this situation in Sect.2. In Sect.3 we will discuss the $\gamma X$ decay channel, with $X$ a scalar particle, and in Sect.4 we will discuss other decays. In Sect.5 we will give our conclusions.

\section{The contribution of $X$ to the effective degrees of freedom}

As we will see, we will be mainly interested in the exotic particle $X$ being a scalar $S$. Let us then consider this case: $X=S$. The scalar particle $S$ was in thermal equilibrium in the early Universe, due to its interaction with electrons, positrons and/or photons. It has an interaction rate $\Gamma$ that can be calculated once this interaction is specified.

As the Universe expands and cools, the interaction rate decreases and $S$ decouples at a time $t_{D}$ and at a temperature $T_{D}$ when

$$
\Gamma=H
$$

where $H$ is the expansion rate. Neglecting the curvature term, a completely justified approximation in the early Universe, $H$ is given by

$$
H^{2}=\frac{8 \pi G}{3} \rho=g_{*}(T) \frac{8 \pi^{3} G}{90} T^{4}
$$

In (2) we have defined the effective degrees of freedom $g_{*}(T)$ (we follow the notation of Kolb and Turner [10]).

As is well known, primordial nucleosynthesis offers a limit on the degrees of freedom $g_{*}$ contributing to the early Universe expansion at $T \simeq 1 \mathrm{MeV}$. It comes from a detailed comparison between the observed abundances of the primordial elements and the predictions of the Big Bang model in the nucleosynthesis era [11]. The limit is very stringent and places 
a bound on the energy density $\rho_{S}$ of $S$ compared to the photon contribution $\rho_{\gamma}$ ]

$$
\Delta g_{*} \equiv \frac{\rho_{S}}{1 / 2 \rho_{\gamma}} \leq 0.5 \quad T \simeq 1 \mathrm{MeV}
$$

The scalar particle $S$ would of course contribute to $g_{*}$. Its energy density is

$$
\rho_{S}=\frac{1}{(2 \pi)^{3}} \int d p 4 \pi p^{2} E f(p, t)
$$

where $E=\sqrt{p^{2}+m^{2}}$. Before decoupling, the particle is in thermal equilibrium and its distribution function $f$ is that of a boson at temperature $T$

$$
f(p, t)=[\exp (E / T)-1]^{-1} \quad T \geq T_{D}
$$

The temperature $T$ is a decreasing function of $t$. After decoupling, the number of $S$ particles is conserved, which is expressed by

$$
f(p, t)=f\left(p_{D}, t_{D}\right) \quad T<T_{D}
$$

where the momentum has been redshifted as $p R=p_{D} R_{D}$, with $R$ the scale factor.

From (5) and (6) we get the expression

$$
f(p, t)=\left[\exp \sqrt{\frac{p^{2}}{\theta^{2}}+\frac{m^{2}}{T_{D}^{2}}}-1\right]^{-1} \quad T<T_{D}
$$

where we have defined the time dependent parameter

$$
\theta=\frac{R_{D}}{R} T_{D}
$$

The parameter $\theta$ cannot be interpreted as the temperature of $S$, unless $S$ is massless. $\theta$ can be calculated using entropy conservation, which involves the function $g_{* S}(T)$, the effective degrees of freedom contributing to the entropy,

$$
g_{* S}(T) R^{3} T^{3}=g_{* S}\left(T_{D}\right) R_{D}^{3} T_{D}^{3}
$$

which gives

$$
\theta=T\left(\frac{g_{* S}(T)}{g_{* S}\left(T_{D}\right)}\right)^{1 / 3}
$$

(Here $S$ refers to entropy).

\footnotetext{
${ }^{1} \mathrm{~A}$ very recent analysis 12 on primordial nucleosynthesis tends to suggest that the limit is even smaller than (3). Our final upper bound on exotic branching ratios would be then more stringent.
} 
We have evaluated $\Delta g_{*}$, as defined in (3), numerically, substituting (7) and (10) into (4) and using the values of $g_{*}(T)$ and $g_{* S}(T)$ calculated using their exact definitions (see for example [10]). Then, in order not to spoil the agreement of the predictions of the Hot Big Bang model with the observed primordial abundances, we impose the experimental limit (3)). In the mass range $0 \leq m \leq 1022 \mathrm{keV}$, the limit translates into the condition that the $S$ species must decouple for all the temperatures in the range

$$
1 \mathrm{MeV} \leq T \leq 100 \mathrm{MeV}
$$

\section{The o-Ps $\rightarrow \gamma X$ decay, with $X=S$ a scalar particle}

We will assume in this section that $C$ and $P$ are conserved. There are three ways in which this decay can then proceed:

(A) $S$ is coupled to electrons. (An equivalent model with $X$ a pseudoscalar particle was considered by Samuel [4].) The o-Ps decays via Fig. (1.A). The exotic coupling is described by a Yukawa-type interaction

$$
\mathcal{L}_{A}=g_{A} \bar{\psi} \psi S
$$

where both here and in the following $\psi$ is the electron field.

(B) $S$ is coupled to photons. The decay proceeds through the diagram Fig. (1.B). The effective Lagrangian describing the new coupling is

$$
\mathcal{L}_{B}=\frac{1}{4} g_{B} F^{\mu \nu} F_{\mu \nu} S
$$

(C) There is a contact term coupling electrons to a photon and $S$ as in Fig. (1.C). The Lagrangian describing the interactions has the gauge-invariant form

$$
\mathcal{L}_{C}=\frac{1}{2} g_{C} \bar{\psi} \sigma^{\mu \nu} \psi F_{\mu \nu} S
$$

Let us start with model (A). We have an exotic particle $S$ of mass $m_{S}$, with the interaction Lagrangian given in (12).

In the early Universe, the scalar $S$ is in thermal equilibrium due to the processes

$$
\begin{aligned}
& \gamma S \rightarrow e^{+} e^{-} \\
& e S \rightarrow e \gamma
\end{aligned}
$$

where $e$ can be either $e^{+}$or $e^{-}$. We have to consider all three processes since their contribution to the interaction rate are of approximately the same magnitude. 
Let us work out the contribution of the process $\gamma S \rightarrow e^{+} e^{-}$in some detail. We are interested in temperatures $3 T \geq 3 T_{D}>>m_{e}, m_{S}$. In this limit, the process has a total cross section given by

$$
\sigma[s]=\frac{\alpha g_{A}^{2}}{s} \ln s / m_{e}^{2}
$$

The corresponding interaction rate (per unit time and per $S$ ) is

$$
\Gamma=n_{\gamma}<\sigma v>
$$

where the number density of photons is given by

$$
n_{\gamma}=2 \frac{\xi(3)}{\pi^{2}} T^{3}
$$

The thermalized quantity $\langle\sigma v>$ appears in the evolution equation of the $S$ number density $n_{S}$ (the superscript $e q$ refers to the distribution in thermal equilibrium)

$$
\dot{n}_{S}+3 H n_{S}=-<\sigma v>n_{\gamma}^{e q}\left[n_{S}-n_{S}^{e q}\right]
$$

One can find approximate expressions for $\langle\sigma v\rangle$ in [13]. We write the expression for this quantity in the relativistic limit we are interested in

$$
<\sigma v>=\frac{1}{n_{\gamma}^{e q} n_{S}^{e q}} \int d n_{\gamma}^{e q} d n_{S}^{e q}(1-\cos \theta) \sigma\left[2 E_{\gamma} E_{S}(1-\cos \theta)\right]
$$

Here $E_{i}$ refers to the energy of the particle $i$ and $\theta$ is the angle between $S$ and $\gamma$. Substituting now the cross section (17) into this expression, one obtains after a straightforward calculation

$$
<\sigma v>=\frac{\pi^{4}}{288 \xi(3)^{2}} \frac{\alpha g_{A}^{2}}{T^{2}}\left\{\ln 4-\frac{1}{4}+2\left(1-\gamma+\frac{6 \dot{\xi}(2)}{\pi^{2}}\right)+2 \ln \frac{T}{m_{e}}\right\}
$$

where $\xi(x)$ is the Riemann $\xi$ function, $\dot{\xi}(x)$ its derivative and $\gamma$ is the Euler constant.

We can now obtain restrictions on $g_{A}$ by requiring that $S$ is decoupled in the temperature range determined in (11). For each $T_{D}$ in this range, we obtain an upper limit on $g_{A}$, and our final result is necessarily the most stringent of them all. It turns out that this numerical limit is obtained at $T_{D}^{A} \simeq 1 \mathrm{MeV}$.

The formulae above actually only take account of the process (15), which is one of the reactions that maintains $S$ in equilibrium. The contributions of the other processes (16) can be worked out along the same lines. If one takes all these processes into account, the upper bound on the coupling $g_{A}$ appearing in the effective Lagrangian (12) is found to be 


$$
g_{A} \leq 5 \times 10^{-10} \equiv g_{A}^{\text {upper }}
$$

where we have defined (for a later purpose) the numerical value of the upper limit as $g_{A}^{u p p e r}$.

With the Lagrangian (12), one can calculate the branching ratio

$$
B_{S}=\frac{\Gamma(\mathrm{oP}-\mathrm{s} \rightarrow \gamma S)}{\Gamma(\mathrm{oP}-\mathrm{s})}=g_{A}^{2} \frac{3}{8\left(\pi^{2}-9\right) \alpha^{2}}\left(1-\frac{m_{S}^{2}}{4 m_{e}^{2}}\right)
$$

This leads directely to one of our main results. Inserting (23) in (24), the following stringent limit is found

$$
B_{S} \leq 2 \times 10^{-15} f_{A}\left(m_{S}\right) \leq 2 \times 10^{-15} \equiv B_{\text {upper }}^{A}
$$

where the function $f_{A}\left(m_{S}\right)=B_{S}\left(m_{S}\right) / B_{S}\left(m_{S}=0\right)=1-m_{S}^{2} / 4 m_{e}^{2} \leq 1$, with $B_{S}$ calculated using (12).

As we discussed at the beginning of this section, the decay o-Ps $\rightarrow \gamma S$ is due to new interactions that can be described by effective Lagrangians. We have presented the consequences of assuming that the exotic decay is due to the new interaction (A). Using (12) we obtained the limit (25) on $B_{S}$. We have performed similar analyses in the cases (B) and (C), using (13) and (14) respectively. We will only quote the value of the limits.

For the interactions (B), we obtain

$$
g_{B} \leq 2 \times 10^{-7} \mathrm{GeV}^{-1} \equiv g_{B}^{\text {upper }}
$$

and

$$
B_{S} \leq 2 \times 10^{-17} f_{B}\left(m_{S}\right) \leq 2 \times 10^{-17} \equiv B_{\text {upper }}^{B}
$$

with $f_{B}\left(m_{S}\right)=\left(1-m_{S}^{2} / 4 m_{e}^{2}\right)^{3} \leq 1$. The limit is now obtained at $T_{D}^{B} \simeq 100 \mathrm{MeV}$.

For the case $(\mathrm{C})$, we obtain

$$
g_{C} \leq 1 \times 10^{-7} \mathrm{GeV}^{-2} \equiv g_{C}^{\text {upper }}
$$

and

$$
B_{S} \leq 1 \times 10^{-22} f_{C}\left(m_{S}\right) \equiv B_{\text {upper }}^{C}
$$

with $f_{C}\left(m_{S}\right)=\left(1-m_{S}^{2} / 4 m_{e}^{2}\right)^{3} \leq 1$. The limit is also obtained at $T_{D}^{C} \simeq 100 \mathrm{MeV}$.

The bound on $B_{S}$ we finally can claim is the least restrictive, i.e., $B_{S} \leq 2 \times 10^{-15}$. 


\section{Other o-Ps decays to exotic particles}

In order to discuss other decays, it will be very useful to understand why we have found that the limit on $B_{S}$ is less restrictive in case $(\mathrm{A})$ than in $(\mathrm{B})$, and why the latter is less restrictive than case $(\mathrm{C})$.

The differences arise from the different dimensions $D$ of the operators appearing in the effective Lagrangians. The operator in (12) has $D=4$, in (13) it has $D=5$, while in (14) it has $D=6$. Consequently, the coupling $g_{A}$ is dimensionless, while $g_{B}$ and $g_{C}$ have dimensions $M^{-1}$ and $M^{-2}$, respectively. It is important to notice that there are two energy scales in our problem. On the one hand, we obtain restrictions at temperatures $T_{D}$, where decoupling is necessary in order not to excessively modify the predicted abundances in the primordial nucleosynthesis. On the other hand, we use these restrictions into the exotic o-Ps decay, that occurs at an energy scale $E \sim m_{e}$. This leads to the following relations among the upper limits on the exotic couplings and on the branching ratios, that are valid up to an order of magnitude:

$$
B_{\text {upper }}^{A} \sim \frac{T_{D}^{A} T_{D}^{B}}{m_{e}^{2}} B_{\text {upper }}^{B} \quad, \quad B_{\text {upper }}^{B} \sim \frac{T_{D}^{B} T_{D}^{C}}{m_{e}^{2}} B_{\text {upper }}^{C}
$$

We can understand from this discussion that the least restrictive limit on $B_{S}$ is in the case that the coupling is dimensionless, as it is in (A). This discussion will be the key to show the main point of this section, namely that all decays involving exotic particles in the final state have at least the upper limit $B_{\text {upper }}^{A}$, corresponding to case (A) above.

The o-Ps $\rightarrow \gamma X$ decay, with $X$ a pseudoscalar particle $P$, is for our purposes completely similar to the decay where $X$ is a scalar particle. There are also three ways in which the decay can proceed, described by the three Lagrangians

$$
\begin{aligned}
\mathcal{L}_{1} & =g_{1} \bar{\psi} \gamma_{5} \psi P \\
\mathcal{L}_{2} & =\frac{1}{8} g_{2} \epsilon_{\alpha \beta \gamma \delta} F^{\alpha \beta} F^{\gamma \delta} P \\
\mathcal{L}_{3} & =\frac{1}{2} g_{3} \bar{\psi} \sigma^{\mu \nu} \gamma_{5} \psi F_{\mu \nu} P
\end{aligned}
$$

The conclusions regarding the upper limits one can obtain on $B_{P}$ are exactly the same as the corresponding cases (A), (B) and (C) above. Thus $B_{\text {upper }}^{A}$ is the least restrictive upper limit we can place on the branching ratio of the decay o-Ps $\rightarrow \gamma P$. One can think of interactions that do not conserve $\mathrm{C}$ and/or $\mathrm{P}$ and leading to o-Ps $\rightarrow \gamma X$, with $X$ a scalar or pseudoscalar particle. The results one finds are again exactly the upper limits that we found in cases (A), (B) and (C). 
Let us now consider the decay o-Ps $\rightarrow \gamma X$, with $X$ a spin one or two particle. The effective Lagrangian describing the exotic interaction contains operators with $D \geq 4$. The least restrictive limit will be obtained when $D=4$, since then the coupling is dimensionless. In fact, since $X$ now has more degrees of freedom that could contribute at the nucleosynthesis era, the decoupling temperature that we obtain is higher than the one we found in the case of only one degree of freedom $(X=S$ or $P$ ). Therefore the limit we obtain is even several times more restrictive than that of case $(\mathrm{A})$. Thus $B_{\text {upper }}^{A}$ has to be seen as a conservative upper limit when $X$ has spin different from zero.

Another decay that has been experimentally investigated is o-Ps $\rightarrow$ "nothing". A possibility here is o-Ps $\rightarrow S V$, with both the scalar $S$ and the vector $V$ independently attached to electrons. Here we have two effective Lagrangians with $D=4$, and two independent dimensionless couplings:

$$
\begin{aligned}
\mathcal{L}_{4} & =g_{4} \bar{\psi} \psi S \\
\mathcal{L}_{5} & =g_{5} \bar{\psi} \gamma^{\mu} \psi V_{\mu}
\end{aligned}
$$

Our arguments apply to the product of these two couplings. We have to take into account that one has now two exotic particles and thus more degrees of freedom, and the decoupling will have to occur at higher temperatures making the bound on the branching ratio $\Gamma$ (o-Ps $\rightarrow$ "nothing") $/ \Gamma(\mathrm{o}-\mathrm{Ps})$ even more stringent than $B_{\text {upper }}^{A}$. Another possibility is to have contact terms like

$$
\begin{aligned}
\mathcal{L}_{6} & =g_{6} \bar{\psi} \gamma^{\mu} \psi V_{\mu} S \\
\mathcal{L}_{7} & =g_{7} \bar{\psi} \sigma^{\mu \nu} \psi\left(\partial_{\mu} V_{\nu}\right) S
\end{aligned}
$$

The coupling $g_{6}$ has dimension $M^{-1}$ and the coupling $g_{7}$ has dimension $M^{-2}$; thus the bound on the branching ratio will be much more restrictive than $B_{u p p e r}^{A}$. There are still other ways to obtain a o-Ps $\rightarrow$ "nothing" decay. We will not list all the possibilities, since it is easy to see that we will always have limits more stringent than $B_{u p p e r}^{A}$.

An interesting decay of o-Ps would be into a photon and two exotic particles. In fact, experiments are optimized to search for a monochromatic photon, so that they are not sensitive to this type of decay. We can apply our arguments based on nucleosynthesis to restrict this possibility. For example, consider o-Ps $\rightarrow \gamma S_{1} S_{2}$. The case that the two scalars are independently attached to electrons is similar to the above mentioned o-Ps $\rightarrow S V$ decay, and one reaches the same conclusion, namely that $B_{\text {upper }}^{A}$ is a conservative upper limit on the branching ratio of the exotic decay. If both scalar particles emerge in a contact term with 
electrons, or if the scalars are coupled to two photons, the coupling is dimensionful and the bound is still more stringent.

The type of arguments discussed in this section can be applied to any decay with exotic particles in the final state. One first has to write the effective Lagrangians that can lead to the decay channel. The order of magnitude of the limit on the branching ratio depends on the dimensions of the coupling, and to a lesser extent on the number of exotic degrees of freedom. The least stringent limit is obtained when the coupling is dimensionless and there is a scalar or pseudoscalar particle in the final state (model (A) or closely related), so that we place the following limit on any exotic decay

$$
B=\frac{\Gamma(\mathrm{o}-\mathrm{Ps} \rightarrow \text { "exotic" }+\ldots)}{\Gamma(\mathrm{o}-\mathrm{Ps})} \leq 2 \times 10^{-15}
$$

\section{Conclusions}

The o-Ps decay puzzle would be solved if there were new decays with branching ratios $\sim 10^{-3}$. Some of these decays have been ruled out by existing laboratory experiments.

In this letter we have shown that all o-Ps decays involving exotic particles are restricted by nucleosynthesis arguments. Using an effective Lagrangian approach, we have been able to place a very stringent upper limit for any exotic decay

$$
B=\frac{\Gamma(\mathrm{o}-\mathrm{Ps} \rightarrow \text { "exotic" }+\ldots)}{\Gamma(\mathrm{o}-\mathrm{Ps})} \leq 2 \times 10^{-15}
$$

Our result excludes the exotic decay solution to the o-Ps puzzle.

\section{Acknowledgements}

We thank the Theoretical Astroparticle Network for support under the EEC Contract No. CHRX-CT93-0120 (Direction Generale 12 COMA). This work has been partially supported by the CICYT Research Project No's. AEN-93-0474 and AEN-93-0520. R.E. acknowledges a FPI Grant from the Universitat Autònoma de Barcelona and R.T. acknowledges a FPI Grant from the Ministeri d'Educació i Ciència (Spain). 


\section{References}

[1] C. I. Westbrook, D. W. Gidley, R. S. Conti and A. Rich, Phys. Rev. Lett. 58 (1987) 1328; Phys. Rev. A40 (1989) 5489.

[2] J. S. Nico, D. W. Gidley, A. Rich and P. W. Zitzewitz, Phys. Rev. Lett. 65 (1990) 1344.

[3] W. E. Caswell and G. P. Lepage, Phys. Rev. A20 (1979) 36;

G. S. Adkins, Ann. Phys. N. Y. 146 (1983) 78.

[4] M. A. Samuel, Mod. Phys. Lett. A Vol. 3 No. 11 (1988) 1117.

[5] T. Maeno et al., UT-ICEPP 95-01, March 1995.

[6] S. Asai, K. Shigekuni, T. Sanuki and S. Orito, Phys. Lett. B323 (1994) 90.

[7] S. Asai, S. Orito, K. Yoshimura and T. Haga, Phys. Rev. Lett. 66 (1991) 2440.

[8] T. Mitsui et al., Phys. Rev. Lett. 70 (1993) 2265.

[9] M. V. Akopyan, G. S. Atoyan, S. N. Gninenko and V. V. Sukhov, Phys. Lett. B272 (1991) 443.

M. Tsuchiaki, S. Orito, T. Yoshida and M. Minowa, Phys. Lett. B236 (1990) 81.

S. Orito et al. Phys. Rev. Lett. 63 (1989) 597.

G. S. Atoyan, S. N. Gninenko, V. I. Razin and Y. V. Ryabov, Phys. Lett. B220 (1989) 317.

[10] E.W. Kolb and M.S. Turner, The Early Universe, Frontiers in Physics, Addison-Wesley, 1990.

[11] T.P. Walker, G. Steigman, D.N. Schramm, K.A. Olive and H. Kang, Astrophys. J. 376 (1991) 51.

[12] N. Hata et al., OSU-TA-6/95, UPR-0654T, May 1995.

[13] J. Bernstein, L.S. Brown and G. Feinberg, Phys. Rev. D32 (1985) 3261.

M. Srednicki, R. Watkins and K.A. Olive, Nucl. Phys. B310 (1988) 693.

P. Gondolo and G. Gelmini, Nucl. Phys. B360 (1991) 145. 


\section{Figure Caption}

Fig.1. Exotic o-Ps decays due to the effective Lagrangians in models (A), (B) or (C). 
$\mathrm{e}^{t}$

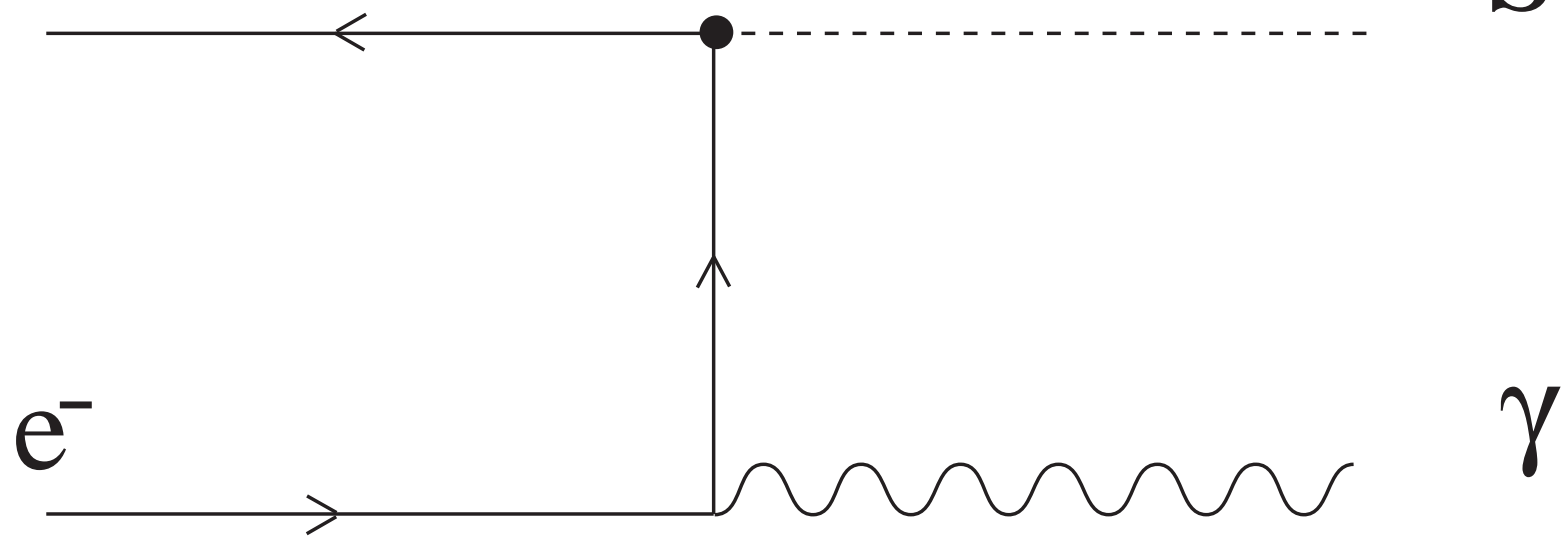

(1.A)

$e^{+}$

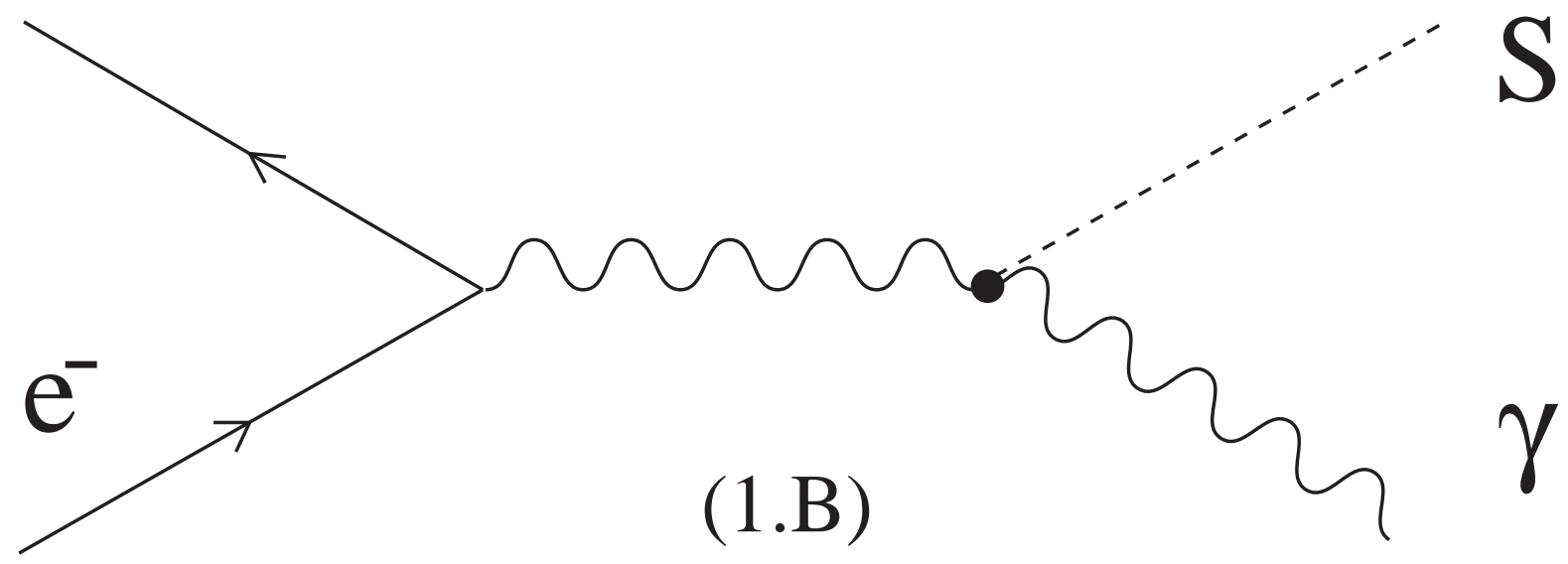

$e^{+}$

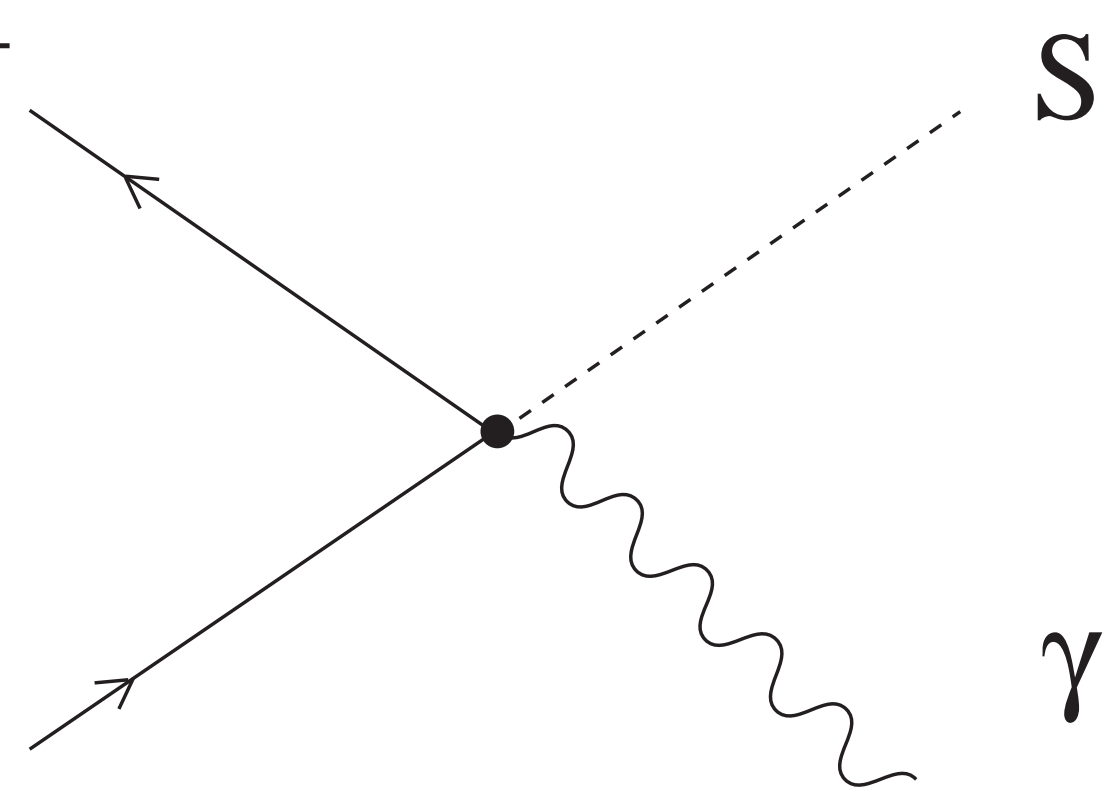

(1.C) 